\title{
Existence, uniqueness, and stability of uncertain delay differential equations with $V$-jump
}

\author{
Zhifu Jia', Xinsheng Liu' ${ }^{1 *}$ and Cunlin Li
}

\section{"Correspondence:}

xsliu@nuaa.edu.cn

${ }^{1}$ State Key Laboratory of Mechanics Control of Mechanical Structures, Institute of Nano Science and Department of Mathematics, Nanjing University of Aeronautics and Astronautics, 210016 Nanjing, China

Full list of author information is available at the end of the article

\begin{abstract}
No previous study has involved uncertain delay differential equations with jump. In this paper, we consider the uncertain delay differential equations with $V$-jump, which is driven by both an uncertain $V$-jump process and an uncertain canonical process. First of all, we give the equivalent integral equation. Next, we establish an existence and uniqueness theorem of solution to the differential equations we proposed in the finite domain and the infinite domain, respectively. Once more, the concept of stability for uncertain delay differential equations with $V$-jump is proposed. In addition, the sufficient condition for stability theorem is derived. To judge existence, uniqueness, and stability briefly, we provide some examples in the end.
\end{abstract}

Keywords: V-jump process; One-sided local Lipschitz condition; Existence and uniqueness; Linear growth condition; Stability

\section{Introduction}

More than half a century ago, when the Itô's [1] landmark work "On stochastic differential equations" (Itô, 1951) came out, the stochastic differential equations (SDEs), as a new branch of mathematics, aroused great interest in academic circles. After more than half a century of glorious development, SDEs are famous all over the world. In recent decades, SDEs have accumulated many results, which played an important role in financial [2], control theory [3], biomathematics [4], game theory [5], and other models hidden in the observed data. It is well known that the essences of SDEs are based on an axiomatic probability theory, and large amounts of sample data are needed to obtain the frequency of their random disturbances. Furthermore, their distribution functions can be obtained. However, in reality, people seem to lack data or the size of sample data applied in practice is smaller in some cases, such as the emerging infectious disease model, the new stock model, and so on. Although sometimes we have a lot of available sample data, the frequency obtained by sample data is, unfortunately, not close enough to the distribution function obtained in some practical problems, and we need to invite some domain experts to evaluate the belief degree that each event may happen in these situations.

Human uncertainty with respect to belief degrees [6] can play an important role in addressing the issue of an indeterminate phenomenon. In order to describe the evolution of

(c) The Author(s) 2020. This article is licensed under a Creative Commons Attribution 4.0 International License, which permits use sharing, adaptation, distribution and reproduction in any medium or format, as long as you give appropriate credit to the original author(s) and the source, provide a link to the Creative Commons licence, and indicate if changes were made. The images or other third party material in this article are included in the article's Creative Commons licence, unless indicated otherwise in a credit line to the material. If material is not included in the article's Creative Commons licence and your intended use is not permitted by statutory regulation or exceeds the permitted use, you will need to obtain permission directly from the copyright holder. To view a copy of this licence, visit http://creativecommons.org/licenses/by/4.0/. 
an uncertain phenomenon with respect to belief degrees, the uncertain differential equations were first proposed by Liu [7]. Following that, Liu [8] also proposed the concept of stability of uncertain differential equations. Later, Chen and Liu [9] proved an existence and uniqueness theorem for an uncertain differential equation, and Yao et al. [10] proved some related stability theorems. Besides, a large and growing body of literature [11-15] about stability theorems for uncertain differential equations has been investigated. Furthermore, Yao and Chen [16] first proposed Euler's method combined with 99-method or 999-method to obtain the numerical solution of the uncertain differential equation. With the perfect theory and maturity of numerical methods of the uncertain differential equation, uncertain differential equations have been successfully applied to many areas such as optimal control [17], differential game theory [18, 19], wave equation [20-22], financial systems [23], fractional differential equations [24], and so on. To better understand the development of uncertain differential equations and applications of numerical methods, the readers can refer to the book [25].

$V$-jump uncertain processes proposed by Deng et al. [26] were often used to describe the evolution of an uncertain phenomenon with jumps, in which the uncertain process may undergo a sudden change because of emergency such as economic crisis, outbreaks of infectious diseases, earthquake, war, etc. The definition of $V$-jump uncertain process is as follows.

Definition 1 An uncertain process $V_{k}$ with respect to time $k$ is said to be a $V$-jump process with parameters $\theta_{1}$ and $\theta_{2}\left(0<\theta_{1}<\theta_{2}<1\right)$ for $k \geq 0$ if

(i) $V_{0}=0$,

(ii) $V_{k}$ has stationary and independent increments,

(iii) for any given time $k>0$, every increment $V_{r+k}-V_{r}$ is a $\mathcal{Z}$ jump uncertain variable $\xi \sim \mathcal{Z}\left(\theta_{1}, \theta_{2}, k\right)$ for $\forall r>0$ whose uncertainty distribution is

$$
\Phi(x)= \begin{cases}0 & \text { if } x<0, \\ \frac{2 \theta_{1}}{k} x & \text { if } 0 \leq x<\frac{k}{2}, \\ \theta_{2}+\frac{2\left(1-\theta_{2}\right)}{k}\left(x-\frac{k}{2}\right) & \text { if } \frac{k}{2} \leq x<k \\ 1 & \text { if } x \geq k\end{cases}
$$

Deng et al. [27] proved the existence and uniqueness of a solution to uncertain differential equation with $V$-jump under Lipschitz condition and linear growth condition on the coefficients. The definition of uncertain differential equation with $V$-jump is as follows.

Definition 2 Suppose that $C_{k}$ is an uncertain canonical process with respect to time $k, V_{k}$ is an uncertain $V$-jump process with respect to time $k$, and $p_{1}, p_{2}$, and $p_{3}$ are some given functions. Then

$$
d Z_{k}=p_{1}\left(Z_{k}, k\right) d k+p_{2}\left(Z_{k}, k\right) d C_{k}+p_{3}\left(Z_{k}, k\right) d V_{k}
$$

is called an uncertain differential equation with $V$-jump.

Uncertain differential equations with $V$-jumps were widely applied to uncertain optimal control with $V$-jumps, see Refs [28-33]; whereas uncertain delay differential equations [34-38] were often used to describe such uncertain physical systems that depend 
not only on the present state but also upon their past states. The main interest in the theory of uncertain delay differential equations was related to the existence, uniqueness as well as stability. Aiming at these phenomena, Barbacioru [34] proposed uncertain delay differential equations. Ge et al. [35] proved the existence and uniqueness of solutions under Lipschitz condition and linear growth condition on the coefficients. Later, Wang et al. $[36,37]$ proposed some concepts of the stability and proved the corresponding stability theorems. Jia and Sheng [38] proved stability in distribution. The definition of uncertain delay differential equation is as follows.

Definition 3 Suppose that $C_{k}$ is a Liu process with respect to time $k$, and $h$ and $p$ are two continuous functions. Then

$$
\begin{cases}d Z_{k}=h\left(k, Z_{k}, Z_{k-\tau}\right) d k+p\left(k, Z_{k}, Z_{k-\tau}\right) d C_{k}, & k \in[0,+\infty), \\ Z_{k}=\varphi(k), & k \in[-\tau, 0]\end{cases}
$$

is called an uncertain delay differential equation, where $\tau$ is called time delay. Its equivalent integral form is as follows:

$$
\begin{cases}Z_{k}=Z_{0}+\int_{0}^{k} h\left(r, Z_{r}, Z_{r-\tau}\right) d r+\int_{0}^{k} p\left(r, Z_{r}, Z_{r-\tau}\right) d C_{r}, & k \in[0,+\infty), \\ Z_{k}=\varphi(k), & k \in[-\tau, 0] .\end{cases}
$$

However, the uncertain delay differential equations with $V$-jump have not been studied so far. For describing the state of an uncertain delay system with jumps more accurately, we propose uncertain delay differential equations with $V$-jump. In contrast to earlier results of Refs [27, 35], we not only combine these two equations, but also prove the existence and uniqueness of solutions by one-sided local Lipschitz condition rather than the strict Lipschitz condition on the coefficients. Furthermore, under some reasonable conditions, we prove the stability.

The remainder of the paper is organized as follows. In Sect. 2, we prove an existence, uniqueness, and stability theorem of the solution to uncertain delay differential equations with $V$-jump and give some examples. Finally, a brief conclusion is given in Sect. 3.

\section{Main results}

We first give the concept of uncertain delay differential equations with $V$-jump and an example.

\subsection{Uncertain delay differential equations with $V$-jump}

Definition 4 Suppose that $C_{k}$ is an uncertain canonical process, $V_{k}$ is an uncertain $V$ jump process with respect to time $k$, and $h(k, z), p(k, z), q(k, z):[0, T] \times \mathbb{R} \rightarrow \mathbb{R}$ are continuous maps. Then

$$
\left\{\begin{array}{rlrl}
d Z_{k}=h\left(k, Z_{k}, Z_{k-\tau}\right) d k+p\left(k, Z_{k}, Z_{k-\tau}\right) d C_{k} & & \\
& +q\left(k, Z_{k}, Z_{k-\tau}\right) d V_{k}, & & k \in[0,+\infty), \\
Z_{k}=\varphi(k), & & k \in[-\tau, 0]
\end{array}\right.
$$


is called an uncertain differential equation with $V$-jump. A solution is an uncertain process $Z_{k}$ that satisfies (3) identically in time $k$. If $\tau$ is finite, the equation with $V$-jump is called the one with finite delay; otherwise, the one with infinite delay.

To solve an uncertain delay differential equations with $V$-jump, we first give an example. Consider the following uncertain delay differential equation with $V$-jump:

$$
\begin{cases}d Z_{k}=m Z_{k-\tau} d k+\mu d C_{k}+v d V_{k}, & k \in[0, T], \\ Z_{k}=1, & k \in[-\tau, 0],\end{cases}
$$

where $\tau>0, m, \mu$, and $v$ are constants.

For $k \in[0, T]$, there exists $n \in \mathbb{N}$ such that $k \in[n \tau,(n+1) \tau]$. If $n=0, k \in[0, \tau]$, then $k-\tau \in[-\tau, 0]$, and $Z_{k-\tau}=1$. Thus, we have

$$
\begin{aligned}
& \left\{\begin{array}{l}
d Z_{k}=m d k+\mu d C_{k}+v d V_{k}, \\
Z_{0}=1,
\end{array}\right. \\
& Z_{k}=Z_{0}+\int_{0}^{k} m d r+\int_{0}^{k} \mu d C_{r}+\int_{0}^{k} v d V_{r}
\end{aligned}
$$

If $n=1, k \in[\tau, 2 \tau]$, then $k-\tau \in[0, \tau]$, and

$$
Z_{k-\tau}=1+m(k-\tau)+\mu C_{k-\tau}+v V_{k-\tau} .
$$

So, we have

$$
\begin{aligned}
\left\{\begin{array}{l}
d Z_{k}=m\left[1+m(k-\tau)+\mu C_{k-\tau}+\nu V_{k-\tau}\right] d k+\mu d C_{k}+v d V_{k} \\
Z_{\tau}
\end{array}\right. & 1+m \tau+\mu C_{\tau}+\nu V_{\tau}, \\
Z_{k}= & Z_{\tau}+\int_{\tau}^{k} m\left[1+m(r-\tau)+\mu C_{r-\tau}\right. \\
& \left.+v V_{r-\tau}\right] d r+\int_{\tau}^{k} \mu d C_{r}+\int_{\tau}^{k} \nu d V_{r} \\
= & 1+m \tau+\mu C_{\tau}+v V_{\tau}+\int_{\tau}^{k} m\left[1+m(r-\tau)+\mu C_{r-\tau}\right. \\
& \left.+v V_{r-\tau}\right] d r+\mu\left(C_{k}-C_{\tau}\right)+v\left(V_{k}-V_{\tau}\right) \\
= & 1+m k+m^{2} \frac{(k-\tau)^{2}}{2}+\mu C_{k}+v V_{k} \\
& +m \mu \int_{\tau}^{k} C_{r-\tau} d r+m v \int_{\tau}^{k} V_{r-\tau} d r .
\end{aligned}
$$

Continuing this method, we can find the expression for $Z(k)$ on each interval $[n \tau,(n+1) \tau]$ with $n \in \mathbb{N}$. 
Remark 1 According to the definition of uncertain canonical $C_{k}(\gamma)$, where $\gamma \in \Gamma$ defined in Definition 2.2 [9], almost all sample paths of $C_{k}$ are Lipschitz continuous functions. That is, there exists a set $\Gamma_{0}$ in $\Gamma$ with $\mathcal{M}\left\{\Gamma_{0}\right\}=1$ such that, for any $\gamma \in \Gamma_{0}, C_{k}(\gamma)$ is Lipschitz continuous. To do this simply, we set $\Gamma_{0}=\Gamma$. Thus, for each $\gamma$, by Lemma 4.1 in [9], there exists a positive number $K(\gamma)$ such that

$$
\left|C_{r}(\gamma)-C_{k}(\gamma)\right| \leq K(\gamma)|r-k|, \quad \forall r, k \geq 0
$$

and for each sample $\gamma$, it follows from the definition of uncertain $V$-jump process and Theorem 3.2 in [27] that

$$
\left|V_{r}(\gamma)-V_{k}(\gamma)\right| \leq|r-k|, \quad \forall r, k \geq 0
$$

Besides, the uncertain integrals of $C_{k}$ and $V_{k}$ are equivalent to the Riemann-Stieltjes integral from the point of each sample path. Hence, we can just focus on the following uncertain delay integral equation with $V$-jump:

$$
\left\{\begin{aligned}
Z_{k}(\gamma)= & Z_{0}(\gamma)+\int_{0}^{k} h\left(r, Z_{r}(\gamma), Z_{r-\tau}(\gamma)\right) d r & & \\
& +\int_{0}^{k} p\left(r, Z_{r}(\gamma), Z_{r-\tau}(\gamma)\right) d C_{r}(\gamma) & & \\
& +\int_{0}^{k} q\left(r, Z_{r}(\gamma), Z_{r-\tau}(\gamma)\right) d V_{r}, & & k \in[0,+\infty), \\
Z_{k}(\gamma)= & \varphi(k), & & k \in[-\tau, 0] .
\end{aligned}\right.
$$

Our goal is to prove that, for each sample path $\gamma$, the uncertain delay integral equation with $V$-jump (7) has a unique solution on $[0,+\infty)$ under certain reasonable conditions.

First of all, we discuss the existence and uniqueness for uncertain delay differential equations with $V$-jump in a local interval $\left[k_{0}, k_{0}+\alpha\right]$ for some positive $\alpha$. Equation (7) becomes

$$
\left\{\begin{aligned}
Z_{k}(\gamma)= & Z_{k_{0}}(\gamma)+\int_{k_{0}}^{k} h\left(r, Z_{r}(\gamma), X_{r-\tau}(\gamma)\right) d r & & \\
& +\int_{k_{0}}^{k} p\left(r, Z_{r}(\gamma), Z_{r-\tau}(\gamma)\right) d C_{r}(\gamma) & & \\
& +\int_{k_{0}}^{k} q\left(r, Z_{r}(\gamma), Z_{r-\tau}(\gamma)\right) d V_{r}, & & k \in\left[k_{0}, k_{0}+\alpha\right], \\
Z_{k}(\gamma)= & \varphi(k), & & k \in\left[k_{0}-\tau, k_{0}\right],
\end{aligned}\right.
$$

and the following Theorem 1 will give the result of existence and uniqueness of uncertain delay integral equation with $V$-jump (8).

\subsection{Existence and uniqueness of the solution}

Theorem 1 Fixing $\gamma \in \Gamma$, the uncertain delay integral equation with $V$-jump (8) has a unique solution in $\left[k_{0}, k_{0}+\alpha\right]$ if the coefficients $h, p$, and $q$ are locally Lipschitz continuous of $z$. In other words, for each

$$
D=\left\{(k, z, \hat{z}) \mid k \in\left[k_{0}, k_{0}+a\right], z \in\left[Z_{k_{0}}(\gamma)-b, Z_{k_{0}}(\gamma)+b\right], \hat{z} \in \mathbb{R}\right\}
$$

there exists a positive constant $L_{D}$ such that

$$
\left|h\left(k, z_{1}, \hat{z}\right)-h\left(k, z_{2}, \hat{z}\right)\right| \vee\left|p\left(k, z_{1}, \hat{z}\right)-p\left(k, z_{2}, \hat{z}\right)\right| \vee\left|q\left(k, z_{1}, \hat{z}\right)-q\left(k, z_{2}, \hat{z}\right)\right| \leq L_{D}\left|z_{1}-z_{2}\right|,
$$


where $a>0, b>0,\left(k, z_{1}, \hat{z}\right) \in D,\left(k, z_{2}, \hat{z}\right) \in D$, and

$$
Q=\max _{D}\{|h(k, z, \hat{z})|+K(\gamma)|p(k, z, \hat{z})|+|q(k, z, \hat{z})|\}
$$

$K(\gamma)$ is the Lipschitz constant to $C_{k}(\gamma)$, and $\alpha=\min \{a, b / Q, \tau\}$.

Proof By using successive approximations, we will prove this theorem in three steps.

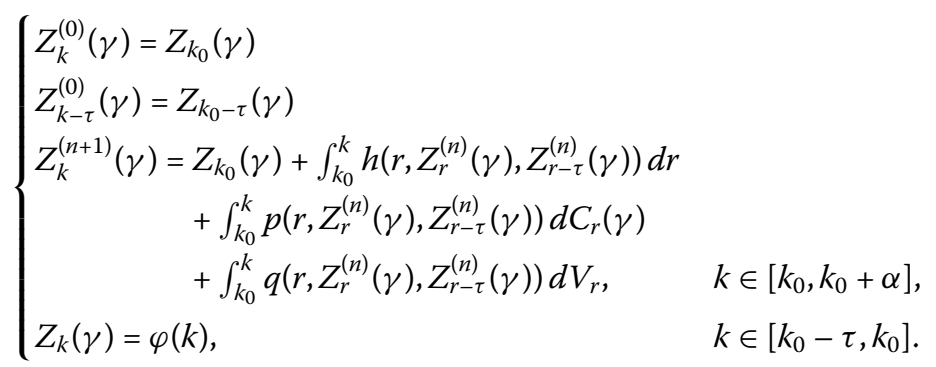

It is easy to find that $\left\{Z_{k}^{(n)}(\gamma)\right\}$ is continuous in time $k$ for any $n \geq 0$.

Step 1. (existence) In this step, we will prove that

$$
\left(k, Z_{k}^{(n)}(\gamma), Z_{k-\tau}^{(n)}(\gamma)\right) \in D, \quad n \geq 0,
$$

when $k \in\left[k_{0}, k_{0}+\alpha\right]$.

Here, we use mathematical induction. When $n=0$,

$$
\left\{\begin{array}{l}
k \in\left[k_{0}, k_{0}+a\right] \\
Z_{k}^{(0)}(\gamma)=Z_{k_{0}}(\gamma) \in\left[Z_{k_{0}}(\gamma)-b, Z_{k_{0}}(\gamma)+b\right] \\
Z_{k-\tau}^{(0)}(\gamma)=Z_{k_{0}-\tau}(\gamma) \in \mathbb{R} .
\end{array}\right.
$$

Thus the conclusion is obviously established. Assume that

$$
\left(k, Z_{k}^{(n)}(\gamma), Z_{k-\tau}^{(n)}(\gamma)\right) \in D, \quad n \geq 0,
$$

when $k \in\left[k_{0}, k_{0}+\alpha\right]$, we have

$$
\begin{aligned}
& \left|Z_{k}^{(n+1)}(\gamma)-Z_{k_{0}}(\gamma)\right| \\
& =\mid \int_{k_{0}}^{k} h\left(r, Z_{r}^{(n)}(\gamma), Z_{r-\tau}^{(n)}(\gamma)\right) d r+\int_{k_{0}}^{k} p\left(r, X_{r}^{(n)}(\gamma), Z_{r-\tau}^{(n)}(\gamma)\right) d C_{r}(\gamma) \\
& \quad+\int_{k_{0}}^{k} q\left(r, Z_{r}^{(n)}(\gamma), Z_{r-\tau}^{(n)}(\gamma)\right) d V_{r} \mid \\
& \leq\left|\int_{k_{0}}^{k} h\left(r, Z_{r}^{(n)}(\gamma), Z_{r-\tau}^{(n)}(\gamma)\right) d r\right|+K(\gamma)\left|\int_{k_{0}}^{k} p\left(r, Z_{r}^{(n)}(\gamma), Z_{r-\tau}^{(n)}(\gamma)\right) d r\right| \\
& \quad+\left|\int_{k_{0}}^{k} q\left(r, Z_{r}^{(n)}(\gamma), Z_{r-\tau}^{(n)}(\gamma)\right) d r\right| \\
& =\int_{k_{0}}^{k}\left|h\left(r, Z_{r}^{(n)}(\gamma), Z_{r-\tau}^{(n)}(\gamma)\right)\right|+K(\gamma)\left|p\left(r, Z_{r}^{(n)}(\gamma), Z_{r-\tau}^{(n)}(\gamma)\right)\right|
\end{aligned}
$$




$$
\begin{aligned}
& +\left|q\left(r, Z_{r}^{(n)}(\gamma), Z_{r-\tau}^{(n)}(\gamma)\right)\right| d r \\
\leq & Q \cdot\left|k-k_{0}\right| \\
\leq & Q \cdot \alpha \leq b,
\end{aligned}
$$

and

$$
Z_{k-\tau}^{(n+1)}(\gamma) \in \mathbb{R}
$$

This indicates that $\left(k, Z_{k}^{(n)}(\gamma), Z_{k-\tau}^{(n)}(\gamma)\right) \in D$ for $n=0,1,2, \ldots$, when $k \in\left[k_{0}, k_{0}+\alpha\right]$.

Step 2. In this step, we will prove that the sequence $\left\{Z_{k}^{(n)}(\gamma)\right\}_{n=0}^{+\infty}$ given by (9) converges uniformly to the solution of equation (8) on $\left[k_{0}, k_{0}+\alpha\right]$ as $n \rightarrow \infty$.

First, we will prove

$$
\left|Z_{k}^{(n+1)}(\gamma)-Z_{k}^{(n)}(\gamma)\right| \leq \frac{Q\left(2 L_{D}+K(\gamma) L_{D}\right)^{n}}{(n+1) !}\left|k-k_{0}\right|^{n+1} .
$$

Similar to Step 1, in this step, we also use mathematical induction. When $n=0$,

$$
\begin{aligned}
\left|Z_{k}^{(1)}(\gamma)-Z_{k}^{(0)}(\gamma)\right| & \\
= & \mid \int_{k_{0}}^{k} h\left(r, Z_{r}^{(0)}(\gamma), Z_{r-\tau}^{(0)}(\gamma)\right) d r+\int_{k_{0}}^{k} p\left(r, Z_{r}^{(0)}(\gamma), Z_{r-\tau}^{(0)}(\gamma)\right) d C_{r}(\gamma) \\
& +\int_{k_{0}}^{k} q\left(r, Z_{r}^{(0)}(\gamma), Z_{r-\tau}^{(0)}(\gamma)\right) d V_{r} \mid \\
= & \left|\int_{k_{0}}^{k} h\left(r, Z_{r}^{(0)}(\gamma), Z_{r-\tau}^{(0)}(\gamma)\right) d r\right|+K(\gamma)\left|\int_{k_{0}}^{k} p\left(r, Z_{r}^{(0)}(\gamma), Z_{r-\tau}^{(0)}(\gamma)\right) d r\right| \\
& +\left|\int_{k_{0}}^{k} q\left(r, Z_{r}^{(0)}(\gamma), Z_{r-\tau}^{(0)}(\gamma)\right) d r\right| \\
\leq & \int_{k_{0}}^{k}\left|h\left(r, Z_{r}^{(0)}(\gamma), Z_{r-\tau}^{(0)}(\gamma)\right)\right|+K(\gamma)\left|p\left(r, Z_{r}^{(0)}(\gamma), Z_{r-\tau}^{(0)}(\gamma)\right)\right| \\
& +\left|q\left(r, Z_{r}^{(0)}(\gamma), Z_{r-\tau}^{(0)}(\gamma)\right)\right| d r \\
\leq & Q \cdot\left|k-k_{0}\right| .
\end{aligned}
$$

Assume that

$$
\begin{aligned}
& \left|Z_{k}^{(n)}(\gamma)-Z_{k}^{(n-1)}(\gamma)\right| \\
& \quad \leq \frac{Q\left(2 L_{D}+K(\gamma) L_{D}\right)^{n-1}}{n !}\left|k-k_{0}\right|^{n},
\end{aligned}
$$

when $k \in\left[k_{0}, k_{0}+\alpha\right]$, we have

$$
\begin{aligned}
& \left|Z_{k}^{(n+1)}(\gamma)-Z_{k}^{(n)}(\gamma)\right| \\
& \quad=\mid \int_{k_{0}}^{k} h\left(r, Z_{r}^{(n)}(\gamma), Z_{r-\tau}^{(n)}(\gamma)\right) d r+\int_{k_{0}}^{k} p\left(r, Z_{r}^{(n)}(\gamma), Z_{r-\tau}^{(n)}(\gamma)\right) d C_{r}(\gamma)
\end{aligned}
$$




$$
\begin{aligned}
& +\int_{k_{0}}^{k} q\left(r, Z_{r}^{(n)}(\gamma), Z_{r-\tau}^{(n)}(\gamma)\right) d V_{r}-\int_{k_{0}}^{k} h\left(r, Z_{r}^{(n-1)}(\gamma), Z_{r-\tau}^{(n-1)}(\gamma)\right) d r \\
& -\int_{k_{0}}^{k} p\left(r, Z_{r}^{(n-1)}(\gamma), Z_{r-\tau}^{(n-1)}(\gamma)\right) d C_{r}(\gamma)-\int_{k_{0}}^{k} q\left(r, Z_{r}^{(n-1)}(\gamma), Z_{r-\tau}^{(n-1)}(\gamma)\right) d V_{r} \mid \\
\leq & \int_{k_{0}}^{k}\left|h\left(r, Z_{r}^{(n)}(\gamma), Z_{r-\tau}^{(n)}(\gamma)\right) d r-\int_{k_{0}}^{k} h\left(r, Z_{r}^{(n-1)}(\gamma), Z_{r-\tau}^{(n-1)}(\gamma)\right)\right| d r \\
& +\int_{k_{0}}^{k}\left|p\left(r, Z_{r}^{(n)}(\gamma), Z_{r-\tau}^{(n)}(\gamma)\right) d C_{r}(\gamma)-\int_{k_{0}}^{k} p\left(r, Z_{r}^{(n-1)}(\gamma), Z_{r-\tau}^{(n-1)}(\gamma)\right)\right| d C_{r}(\gamma) \\
& +\int_{k_{0}}^{k}\left|q\left(r, Z_{r}^{(n)}(\gamma), Z_{r-\tau}^{(n)}(\gamma)\right) d V_{r}-\int_{k_{0}}^{k} q\left(r, Z_{r}^{(n-1)}(\gamma), Z_{r-\tau}^{(n-1)}(\gamma)\right)\right| d V_{r} \\
\leq & \int_{k_{0}}^{k} L_{D}\left|Z_{r}^{(n)}(\gamma)-Z_{r}^{(n-1)}(\gamma)\right| d r+K(\gamma) \int_{k_{0}}^{k} L_{D}\left|Z_{r}^{(n)}(\gamma)-Z_{r}^{(n-1)}(\gamma)\right| d r \\
& +\int_{k_{0}}^{k} L_{D}\left|Z_{r}^{(n)}(\gamma)-Z_{r}^{(n-1)}(\gamma)\right| d r \\
\leq & L_{D}(2+K(\gamma)) \int_{k_{0}}^{k}\left|Z_{r}^{(n)}(\gamma)-Z_{r}^{(n-1)}(\gamma)\right| d r \\
\leq & L_{D}(2+K(\gamma)) \int_{k_{0}}^{k} \frac{Q\left(2 L_{D}+K(\gamma) L_{D}\right)^{n-1}\left|k-k_{0}\right|^{n} d r}{n !} \\
\leq & \frac{Q\left(2 L_{D}+K(\gamma) L_{D}\right)^{n}}{n !} \int_{k_{0}}^{k}\left|r-k_{0}\right|^{n} d r \\
\leq & \frac{Q\left(2 L_{D}+K(\gamma) L_{D}\right)^{n}\left|k-k_{0}\right|^{n+1} .}{(n+1) !}
\end{aligned}
$$

The above inequality gives an upper bound of

$$
\left|Z_{k}^{(n+1)}(\gamma)-Z_{k}^{(n)}(\gamma)\right|
$$

on $\left[k_{0}, k_{0}+\alpha\right]$ for $n=0,1,2, \ldots$ Obviously, for any $\epsilon>0$, there exists an integer $\mathrm{N}(N>0)$ such that

$$
\begin{aligned}
& \sum_{n \geq N}\left|Z_{k}^{(n+1)}(\gamma)-Z_{k}^{(n)}(\gamma)\right| \\
& \quad \leq \sum_{n \geq N} \frac{Q\left(2 L_{D}+K(\gamma) L_{D}\right)^{n}}{(n+1) !}\left|k-k_{0}\right|^{n+1} \\
& \quad=\frac{Q}{2 L_{D}+K(\gamma) L_{D}} \sum_{n \geq N} \frac{\left(2 L_{D}+K(\gamma) L_{D}\right)^{n+1}}{(n+1) !}\left|k-k_{0}\right|^{n+1} \\
& \quad \leq \frac{Q}{2 L_{D}+K(\gamma) L_{D}} \sum_{n \geq N} \frac{\left(2 L_{D}+K(\gamma) L_{D}\right)^{n+1}}{(n+1) !} \alpha^{n+1} \\
& \quad \leq \frac{Q}{2 L_{D}+K(\gamma) L_{D}} \sum_{n \geq N} \frac{\left(\alpha L_{D}(2+K(\gamma))\right)^{n+1}}{(n+1) !} \\
& \quad<\epsilon
\end{aligned}
$$


where the last inequality from

$$
\lim _{n \rightarrow+\infty} \frac{a^{n+1}}{(n+1) !}=0
$$

Because

$$
Z_{k}^{n}(\gamma)=Z_{k}^{0}(\gamma)+\sum_{i=1}^{n}\left(Z_{k}^{i}(\gamma)-Z_{k}^{i-1}(\gamma)\right)
$$

the above inequality indicates that $Z_{k}^{n}(\gamma)$ converges uniformly on $\left[k_{0}, k_{0}+\alpha\right]$ as $n \rightarrow+\infty$.

Thus, we have

$$
\begin{aligned}
Z_{k}^{(n+1)}(\gamma)= & Z_{k_{0}}(\gamma)+\int_{k_{0}}^{k} h\left(r, Z_{r}^{(n)}(\gamma), Z_{r-\tau}^{(n)}(\gamma)\right) d r \\
& +\int_{k_{0}}^{k} p\left(r, Z_{r}^{(n)}(\gamma), Z_{r-\tau}^{(n)}(\gamma)\right) d C_{r}(\gamma) \\
& +\int_{k_{0}}^{k} q\left(r, Z_{r}^{(n)}(\gamma), Z_{r-\tau}^{(n)}(\gamma)\right) d V_{r}(\gamma) .
\end{aligned}
$$

Denote $Z_{k}(\gamma)=\lim _{n \rightarrow+\infty} Z_{k}^{n}(\gamma)$. Taking the limit on both sides of the above equation, it holds that

$$
\begin{aligned}
Z_{k}(\gamma)= & Z_{k_{0}}(\gamma)+\int_{k_{0}}^{k} h\left(r, Z_{r}(\gamma), Z_{r-\tau}(\gamma)\right) d r \\
& +\int_{k_{0}}^{k} p\left(r, Z_{r}(\gamma), Z_{r-\tau}(\gamma)\right) d C_{r}(\gamma)+\int_{k_{0}}^{k} q\left(r, Z_{r}(\gamma), Z_{r-\tau}(\gamma)\right) d V_{r}(\gamma) .
\end{aligned}
$$

That is, the sequence $\left\{Z_{k}^{n}(\gamma)\right\}$ given by (9) converges uniformly to the solution of equation (8) on $\left[k_{0}, k_{0}+\alpha\right]$ as $n \rightarrow+\infty$.

Because each $\left\{Z_{k}^{n}(\gamma)\right\}$ is continuous, $Z_{k}(\gamma)$ is also continuous on $\left[k_{0}, k_{0}+\alpha\right]$. The proof of existence is completed.

Step 3. (uniqueness) Step 3 will prove that $Z_{k}(\gamma)$ obtained in Step 2 is the unique solution of equation (8) on $\left[k_{0}, k_{0}+\alpha\right]$.

Assume that $\tilde{Z}_{k}(\gamma)$ is another solution of equation (8), i.e.,

$$
\left\{\begin{aligned}
\tilde{Z}_{k}(\gamma)= & Z_{k_{0}}(\gamma)+\int_{k_{0}}^{k} h\left(r, \tilde{Z}_{r}(\gamma), \tilde{Z}_{r-\tau}(\gamma)\right) d r & & \\
& +\int_{k_{0}}^{k} p\left(r, \tilde{Z}_{r}(\gamma), \tilde{Z}_{r-\tau}(\gamma)\right) d C_{r}(\gamma) & & \\
& +\int_{k_{0}}^{k} q\left(r, \tilde{Z}_{r}(\gamma), \tilde{Z}_{r-\tau}(\gamma)\right) d V_{r}, & & k \in\left[k_{0}, k_{0}+\beta\right], \\
\tilde{Z}_{k}(\gamma)= & \varphi(k), & & k \in\left[k_{0}-\tau, k_{0}\right],
\end{aligned}\right.
$$

where $0<\beta \leq \alpha$.

Following the local Lipschitz condition, we have

$$
\begin{aligned}
& \left|Z_{k}(\gamma)-\tilde{Z}_{k}(\gamma)\right| \\
& \quad=\mid \int_{k_{0}}^{k} h\left(r, Z_{r}(\gamma), Z_{r-\tau}(\gamma)\right) d r+\int_{k_{0}}^{k} p\left(r, Z_{r}(\gamma), Z_{r-\tau}(\gamma)\right) d C_{r}(\gamma)
\end{aligned}
$$




$$
\begin{aligned}
& +\int_{k_{0}}^{k} q\left(r, Z_{r}(\gamma), Z_{r-\tau}(\gamma)\right) d V_{r}-\int_{k_{0}}^{k} h\left(r, \tilde{Z}_{r}(\gamma), \tilde{Z}_{r-\tau}(\gamma)\right) d r \\
& -\int_{k_{0}}^{k} p\left(r, \tilde{Z}_{r}(\gamma), \tilde{Z}_{r-\tau}(\gamma)\right) d C_{r}(\gamma)-\int_{k_{0}}^{k} q\left(r, \tilde{Z}_{r}(\gamma), \tilde{Z}_{r-\tau}(\gamma)\right) d V_{r} \mid \\
\leq & \left|\int_{k_{0}}^{k} h\left(r, Z_{r}(\gamma), Z_{r-\tau}(\gamma)\right) d r-\int_{k_{0}}^{k} h\left(r, \tilde{Z}_{r}(\gamma), \tilde{Z}_{r-\tau}(\gamma)\right) d r\right| \\
& +\left|\int_{k_{0}}^{k} p\left(r, Z_{r}(\gamma), Z_{r-\tau}(\gamma)\right) d C_{r}(\gamma)-\int_{k_{0}}^{k} p\left(r, \tilde{Z}_{r}(\gamma), \tilde{Z}_{r-\tau}(\gamma)\right) d C_{r}(\gamma)\right| \\
& +\left|\int_{k_{0}}^{k} q\left(r, Z_{r}(\gamma), Z_{r-\tau}(\gamma)\right) d V_{r}-\int_{k_{0}}^{k} q\left(r, \tilde{Z}_{r}(\gamma), \tilde{Z}_{r-\tau}(\gamma)\right) d V_{r}\right| \\
\leq & \int_{k_{0}}^{k} L_{D}\left|Z_{r}(\gamma)-\tilde{Z}_{r}(\gamma)\right| d r+K(\gamma) \int_{k_{0}}^{k} L_{D}\left|Z_{r}(\gamma)-\tilde{Z}_{r}(\gamma)\right| d r \\
& +\int_{k_{0}}^{k} L_{D}\left|Z_{r}(\gamma)-\tilde{Z}_{r}(\gamma)\right| d r \\
= & L_{D}(2+K(\gamma)) \int_{k_{0}}^{k}\left|Z_{r}(\gamma)-\tilde{Z}_{r}(\gamma)\right| d r .
\end{aligned}
$$

By Gronwall's inequality of [39], we have

$$
\left|Z_{k}(\gamma)-\tilde{Z}_{k}(\gamma)\right| \leq 0 \cdot \exp \left(k\left(L_{D}(2+K(\gamma))\right)\right)=0
$$

That is to say, $Z_{k}(\gamma)=\tilde{Z}_{k}(\gamma)$ for any $\left[k_{0}, k_{0}+\alpha\right]$. The proof of uniqueness is completed. Until now, we have completed the proof of Theorem 1 .

According to Theorem 1, the uncertain delay integral equation with $V$-jump (8) has a unique solution on the local interval $\left[k_{0}, k_{0}+\alpha\right]$. Next, Theorem 2 will prove that the solution of uncertain delay integral equation with $V$-jump (8) can be extended to the infinite domain $[0,+\infty)$.

Theorem 2 Fixing $\gamma \in \Gamma$, the uncertain delay integral equation with $V$-jump (8) has a unique solution on $[0,+\infty)$ if the coefficients $h, p$, and $q$ satisfy one-sided local Lipschitz condition of Theorem 1 and the local linear growth condition. In other words, for each $T>0$, there exists a constant $M_{T}$ such that

$$
\begin{aligned}
& |h(k, z, \hat{z})| \vee|p(k, z, \hat{z})| \vee|q(k, z, \hat{z})| \\
& \quad \leq M_{T}(1+|z|+|\hat{z}|), \quad \forall z, \hat{z} \in \mathbb{R}, k \in[0, T] .
\end{aligned}
$$

Proof Define $\rho=\{k \mid$ uncertain delay integral equation with $V$-jump (7) has a unique continuous solution on $[0, k)\}$, and $\rho=\sup \varrho$. According to Theorem 1 , the set $\rho$ is nonempty.

We will prove that $\rho=+\infty$. Assume that $\rho<+\infty$. By the definition, $Z_{k}(\gamma)$ is the unique solution of equation $(7)$ on $[0, \rho)$. Then we have

$$
\begin{aligned}
& \left|Z_{k}(\gamma)\right|+\left|Z_{k-\tau}(\gamma)\right| \\
& \quad=\mid Z_{0}(\gamma)+\int_{0}^{k} h\left(r, Z_{r}(\gamma), Z_{r-\tau}(\gamma)\right) d r+\int_{0}^{k} p\left(r, Z_{r}(\gamma), Z_{r-\tau}(\gamma)\right) d C_{r}(\gamma)
\end{aligned}
$$


Ja et al. Advances in Difference Equations

(2020)

$2020: 440$

Page 11 of 21

$$
\begin{aligned}
& +\int_{0}^{k} q\left(r, Z_{r}(\gamma), Z_{r-\tau}(\gamma)\right) d V_{r}|+| Z_{-\tau}(\gamma)+\int_{-\tau}^{k-\tau} h\left(r, Z_{r}(\gamma), Z_{r-\tau}(\gamma)\right) d r \\
& +\int_{-\tau}^{k-\tau} p\left(r, Z_{r}(\gamma), Z_{r-\tau}(\gamma)\right) d C_{r}(\gamma)+\int_{-\tau}^{k-\tau} q\left(r, Z_{r}(\gamma), Z_{r-\tau}(\gamma)\right) d V_{r} \mid \\
& \leq\left|Z_{0}(\gamma)\right|+\left|\int_{0}^{k} h\left(r, Z_{r}(\gamma), Z_{r-\tau}(\gamma)\right) d r\right|+K(\gamma)\left|\int_{0}^{k} p\left(r, Z_{r}(\gamma), Z_{r-\tau}(\gamma)\right) d r\right| \\
& +\left|\int_{0}^{k} q\left(r, Z_{r}(\gamma), Z_{r-\tau}(\gamma)\right) d r\right|+\left|Z_{-\tau}(\gamma)\right|+\left|\int_{-\tau}^{k-\tau} h\left(r, Z_{r}(\gamma), Z_{r-\tau}(\gamma)\right) d r\right| \\
& +K(\gamma)\left|\int_{-\tau}^{k-\tau} p\left(r, Z_{r}(\gamma), Z_{r-\tau}(\gamma)\right) d r\right|+\left|\int_{-\tau}^{k-\tau} q\left(r, Z_{r}(\gamma), Z_{r-\tau}(\gamma)\right) d r\right| \\
& =\left|Z_{0}(\gamma)\right|+\left|Z_{-\tau}(\gamma)\right|+\mid \int_{-\tau}^{0} h\left(r, Z_{r}(\gamma), Z_{r-\tau}(\gamma)\right)+K(\gamma) p\left(r, Z_{r}(\gamma), Z_{r-\tau}(\gamma)\right) \\
& +q\left(r, Z_{r}(\gamma), Z_{r-\tau}(\gamma)\right) d r|+| \int_{0}^{k-\tau} h\left(r, Z_{r}(\gamma), Z_{r-\tau}(\gamma)\right) \\
& +K(\gamma) p\left(r, Z_{r}(\gamma), Z_{r-\tau}(\gamma)\right)+q\left(r, Z_{r}(\gamma), Z_{r-\tau}(\gamma)\right) d r \mid \\
& +\left|\int_{0}^{k} h\left(r, Z_{r}(\gamma), Z_{r-\tau}(\gamma)\right)+K(\gamma) p\left(r, Z_{r}(\gamma), Z_{r-\tau}(\gamma)\right)+q\left(r, Z_{r}(\gamma), Z_{r-\tau}(\gamma)\right) d r\right| \\
& \leq\left|Z_{0}(\gamma)\right|+\left|Z_{-\tau}(\gamma)\right|+\Delta+M_{\rho}(2+K(\gamma)) \int_{0}^{k-\tau} 1+\left|Z_{r}(\gamma)\right|+\left|Z_{r-\tau}(\gamma)\right| d r \\
& +M_{\rho}(2+K(\gamma)) \int_{0}^{k} 1+\left|Z_{r}(\gamma)\right|+\left|Z_{r-\tau}(\gamma)\right| d r \\
& \leq\left|Z_{0}(\gamma)\right|+\left|Z_{-\tau}(\gamma)\right|+\Delta+2 \rho M_{\rho}(2+K(\gamma)) \\
& +2 M_{\rho}(2+K(\gamma)) \int_{0}^{k}\left|Z_{r}(\gamma)\right|+\left|Z_{r-\tau}(\gamma)\right| d r \\
& +K^{\prime}(\gamma)
\end{aligned}
$$

for any $k \in[0, \rho)$, where

$$
\Delta=\left|\int_{-\tau}^{0} h\left(r, Z_{r}(\gamma), Z_{r-\tau}(\gamma)\right)+K(\gamma) p\left(r, Z_{r}(\gamma), Z_{r-\tau}(\gamma)\right)+q\left(r, Z_{r}(\gamma), Z_{r-\tau}(\gamma)\right) d r\right| .
$$

Set

$$
A=\left|Z_{0}(\gamma)\right|+\left|Z_{-\tau}(\gamma)\right|+\Delta+2 \rho M_{\rho}(2+K(\gamma)) .
$$

By Gronwall's inequality [39], we have

$$
\left|Z_{k}(\gamma)\right|+\left|Z_{k-\tau}(\gamma)\right| \leq A \cdot \exp \left(2 M_{\rho}(2+K(\gamma))\right) \rho=N_{0}<+\infty, \quad \forall k \in[0, \rho) .
$$

That is to say, $\left|Z_{k}(\gamma)\right|+\left|Z_{k-\tau}(\gamma)\right|$ is bounded on $[0, \rho)$.

Thus, we have

$$
\begin{aligned}
& \left|Z_{k_{1}}(\gamma)-Z_{k_{2}}(\gamma)\right| \\
& \quad=\mid Z_{0}(\gamma)+\int_{0}^{k_{1}} h\left(r, Z_{r}(\gamma), Z_{r-\tau}(\gamma)\right) d r+\int_{0}^{k_{1}} p\left(r, Z_{r}(\gamma), Z_{r-\tau}(\gamma)\right) d C_{r}(\gamma)
\end{aligned}
$$




$$
\begin{aligned}
& +\int_{0}^{k_{1}} q\left(r, Z_{r}(\gamma), Z_{r-\tau}(\gamma)\right) d V_{r}-Z_{0}(\gamma)-\int_{0}^{k_{2}} h\left(r, Z_{r}(\gamma), Z_{r-\tau}(\gamma)\right) d r \\
& -\int_{0}^{k_{2}} p\left(r, Z_{r}(\gamma), Z_{r-\tau}(\gamma)\right) d C_{r}(\gamma)-\int_{0}^{k_{2}} q\left(r, Z_{r}(\gamma), Z_{r-\tau}(\gamma)\right) d V_{r} \mid \\
\leq & \left|\int_{k_{1}}^{k_{2}} h\left(r, Z_{r}(\gamma), Z_{r-\tau}(\gamma)\right) d r\right|+\left|\int_{k_{1}}^{k_{2}} p\left(r, Z_{r}(\gamma), Z_{r-\tau}(\gamma)\right) d C_{r}(\gamma)\right| \\
& +\left|\int_{k_{1}}^{k_{2}} q\left(r, Z_{r}(\gamma), Z_{r-\tau}(\gamma)\right) d V_{r}\right| \\
\leq & \int_{k_{1}}^{k_{2}}\left|h\left(r, Z_{r}(\gamma), Z_{r-\tau}(\gamma)\right)\right| d r+K(\gamma) \int_{k_{1}}^{k_{2}}\left|p\left(r, Z_{r}(\gamma), Z_{r-\tau}(\gamma)\right)\right| d r \\
& +\int_{k_{1}}^{k_{2}}\left|q\left(r, Z_{r}(\gamma), Z_{r-\tau}(\gamma)\right)\right| d r \\
\leq & \left.M_{\rho} \int_{k_{1}}^{k_{2}} 1+\left|Z_{r}(\gamma)\right|+\mid Z_{r-\tau}(\gamma)\right) \mid d r \\
& \left.+M_{\rho} K(\gamma) \int_{k_{1}}^{k_{2}} 1+\left|Z_{r}(\gamma)\right|+\mid Z_{r-\tau}(\gamma)\right) \mid d r \\
& \left.+M_{\rho} \int_{k_{1}}^{k_{2}} 1+\left|Z_{r}(\gamma)\right|+\mid Z_{r-\tau}(\gamma)\right) \mid d r \\
= & \left.M_{\rho}(2+K(\gamma)) \int_{k_{1}}^{k_{2}} 1+\left|Z_{r}(\gamma)\right|+\mid Z_{r-\tau}(\gamma)\right) \mid d r \\
\leq & M_{\rho}(2+K(\gamma)) \int_{k_{1}}^{k_{2}}\left(1+N_{0}\right) d r \\
\leq & M_{\rho}(2+K(\gamma))\left(1+N_{0}\right)\left|k_{1}-k_{2}\right|, \forall k_{1}, k_{2} \in[0, \rho) .
\end{aligned}
$$

It holds that $\lim _{k \rightarrow \rho^{-}} Z_{k}(\gamma)$ exists. Set $Z_{\rho}(\gamma)=\lim _{k \rightarrow \rho^{-}} Z_{k}(\gamma)$. Thus $Z_{k}(\gamma)$ is continuous on the interval $[0, \rho]$, and

$$
\left\{\begin{aligned}
Z_{k}(\gamma)= & Z_{0}(\gamma)+\int_{0}^{k} h\left(r, Z_{r}(\gamma), Z_{r-\tau}(\gamma)\right) d r & & \\
& +\int_{0}^{k} p\left(r, Z_{r}(\gamma), Z_{r-\tau}(\gamma)\right) d C_{r}(\gamma) & & \\
& +\int_{0}^{k} q\left(r, Z_{r}(\gamma), Z_{r-\tau}(\gamma)\right) d V_{r}, & & k \in[0, \rho], \\
Z_{k}(\gamma)= & \varphi(k), & & k \in[-\tau, 0] .
\end{aligned}\right.
$$

Consider the following uncertain delay integral equation with $V$-jump:

$$
\left\{\begin{aligned}
Z_{k}(\gamma)= & Z_{\rho}(\gamma)+\int_{\rho}^{k} h\left(r, Z_{r}(\gamma), Z_{r-\tau}(\gamma)\right) d r & & \\
& +\int_{\rho}^{k} p\left(r, Z_{r}(\gamma), Z_{r-\tau}(\gamma)\right) d C_{r}(\gamma) & & \\
& +\int_{\rho}^{k} q\left(r, Z_{r}(\gamma), Z_{r-\tau}(\gamma)\right) d V_{r}, & & k \in(\rho,+\infty), \\
Z_{k}(\gamma)= & Z_{0}(\gamma)+\int_{0}^{k} h\left(r, Z_{r}(\gamma), Z_{r-\tau}(\gamma)\right) d r & & \\
& +\int_{0}^{k} p\left(r, Z_{r}(\gamma), Z_{r-\tau}(\gamma)\right) d C_{r}(\gamma) & & \\
& +\int_{0}^{k} q\left(r, Z_{r}(\gamma), Z_{r-\tau}(\gamma)\right) d V_{r}, & & k \in[0, \rho], \\
Z_{k}(\gamma)= & \varphi(k), & & k \in[-\tau, 0] .
\end{aligned}\right.
$$


Theorem 1 means that there exists a positive number $\alpha$ such that uncertain delay with $V$-jump integral equation (13) has a unique continuous solution $\tilde{Z}_{k}(\gamma)$ on the interval $[\rho, \rho+\alpha]$.

Thus, setting the function

$$
\hat{Z}_{k}(\gamma)= \begin{cases}Z_{k}(\gamma), & \text { if } k \in[0, \rho], \\ \tilde{Z}_{k}(\gamma), & \text { if } k \in(\rho, \rho+\alpha],\end{cases}
$$

$\hat{Z}_{k}(\gamma)$ is the unique continuous solution of equation (8) on the interval $[0, \rho+\alpha]$. We get a contradiction from $\rho=\sup \varrho<+\infty$. So, $\rho=+\infty$, and the solution of uncertain delay integral equation with $V$-jump (7) can be extended uniquely to $[0,+\infty)$. So we complete the proof of Theorem 2 .

Remark 2 When the functions $h, p$, and $q$ in the uncertain delay differential equation with $V$-jump (3) are independent with the present state $Z_{k}$, then (3) is written as

$$
\left\{\begin{array}{rlrl}
d Z_{k}=h\left(k, Z_{k-\tau}\right) d k+p\left(k, Z_{k-\tau}\right) d C_{k} & & \\
& +q\left(k, Z_{k-\tau}\right) d V_{k}, & & k \in[0,+\infty), \\
Z_{k}(\gamma) & =\varphi(k), & & k \in[-\tau, 0] .
\end{array}\right.
$$

For the uncertain delay differential equation with $V$-jump (15), it is not difficult to find that

$$
Z_{k}=Z_{0}+\int_{0}^{k} h\left(r, Z_{r-\tau}\right) d r+\int_{0}^{k} p\left(r, Z_{r-\tau}\right) d C_{r}+\int_{0}^{k} q\left(r, Z_{r-\tau}\right) d V_{r}
$$

for any $0 \leq k \leq \tau$

Then, for $\tau \leq k \leq 2 \tau$, we have

$$
Z_{k}=Z_{\tau}+\int_{\tau}^{k} h\left(r, Z_{r-\tau}\right) d r+\int_{\tau}^{k} p\left(r, Z_{r-\tau}\right) d C_{r}+\int_{\tau}^{k} q\left(r, Z_{r-\tau}\right) d V_{r} .
$$

Repeat this procedure over the intervals $[2 \tau, 3 \tau],[3 \tau, 4 \tau]$, etc. Finally, we can obtain the explicit solution of uncertain delay differential equation with $V$-jump (15).

\subsection{Stability of the solution}

Definition 5 The uncertain delay differential equation with $V$-jump (8) is said to be stable in measure if, for any two solutions $Z_{k}$ and $\hat{Z}_{k}$ with different initial states,respectively, we have

$$
\lim _{\sup _{r \in[-\tau, 0]}\left|Z_{r}-\hat{Z}_{r}\right| \rightarrow 0} \mathcal{M}\left\{\left|Z_{k}(\gamma)-\hat{Z}_{k}(\gamma)\right|>\epsilon\right\}=0, \quad \forall k>0
$$

for any given number $\epsilon>0$, where $\mathcal{M}$ is uncertain measure.

To illustrate the concept of stability, we first give an example. Consider the following uncertain delay differential equation with $V$-jump:

$$
d Z_{k}=a Z_{k-\tau} d k+b d C_{k}+c d V_{k}, \quad k \in[0,+\infty) .
$$


Obviously, the coefficients $h(k, z, \hat{z})=a \hat{z}, p(k, z, \hat{z})=b$, and $q(k, z, \hat{z})=c$ are one-sided local Lipschitz continuous.

$$
\begin{aligned}
& |h(k, z, \hat{z})| \vee|p(k, z, \hat{z})| \vee|q(k, z, \hat{z})|=|a \hat{z}| \vee|b| \vee|c| \\
& \quad=\max (|a|,|b|,|c|)(1+|z|+|\hat{z}|), \quad \forall z, \hat{z} \in \mathbb{R}, k \in[0, T] .
\end{aligned}
$$

By using Theorem 2, it has a unique continuous solution. We can get that $Z_{k}$ and $\hat{Z}_{k}$ are two solutions of (17) with different initial states $\varphi(k)$ and $\psi(k)(k \in[-\tau, 0])$, respectively.

$$
Z_{k}= \begin{cases}\varphi(k), & k \in[-\tau, 0], \\ \varphi(0)+a \int_{0}^{k} \varphi(r-\tau) d r+b C_{k}+c V_{k}, & k \in(0, \tau], \\ Z_{\tau}+a \int_{\tau}^{t} Z_{r-\tau} d r+b\left(C_{k}-C_{\tau}\right)+c\left(V_{k}-V_{\tau}\right), & k \in(\tau, 2 \tau] \\ \cdots \cdots & \end{cases}
$$

and

$$
\hat{Z}_{k}= \begin{cases}\psi(k), & k \in[-\tau, 0], \\ \psi(0)+a \int_{0}^{k} \psi(r-\tau) d r+b C_{k}+c V_{k}, & k \in(0, \tau], \\ \hat{Z}_{\tau}+a \int_{\tau}^{k} \hat{Z}_{r-\tau} d r+b\left(C_{k}-C_{\tau}\right)+c\left(V_{k}-V_{\tau}\right), & k \in(\tau, 2 \tau], \\ \cdots \cdots & \end{cases}
$$

respectively.

Then

$$
\left|Z_{k}-\hat{Z}_{k}\right|= \begin{cases}|\varphi(k)-\psi(k)|, & k \in[-\tau, 0], \\ |\varphi(0)-\psi(0)|+a \int_{0}^{k}|\psi(r-\tau)-\psi(r-\tau)| d r, & k \in(0, \tau], \\ \left|Z_{\tau}-\hat{Z}_{\tau}\right|+a \int_{\tau}^{k}\left|Z_{r-\tau}-\hat{Z}_{r-\tau}\right| d r, & k \in(\tau, 2 \tau] \\ \cdots \cdots & \end{cases}
$$

Therefore,

$$
\lim _{\sup _{r \in[-\tau, 0]}\left|Z_{r}-\hat{Z}_{r}\right| \rightarrow 0} \mathcal{M}\left\{\left|Z_{k}(\gamma)-\hat{Z}_{k}(\gamma)\right|>\epsilon\right\}=0, \quad \forall k>0
$$

for any given number $\epsilon>0$, and the uncertain delay differential equation with $V$-jump (17) is stable in measure by Definition 5 .

Theorem 3 Assume that the uncertain delay differential equation with $V$-jump (3) has a unique solution for each given initial state. Then it is stable in measure if the coefficients $h(k, z, \hat{z}), p(k, z, \hat{z})$, and $q(k, z, \hat{z})$ satisfy

$$
\begin{aligned}
& \left|h\left(k, z_{1}, \hat{z}\right)-h\left(k, z_{2}, \hat{z}\right)\right| \vee\left|p\left(k, z_{1}, \hat{z}\right)-p\left(k, z_{2}, \hat{z}\right)\right| \vee\left|q\left(k, z_{1}, \hat{z}\right)-q\left(k, z_{2}, \hat{z}\right)\right| \\
& \quad \leq N_{k}\left|z_{1}-z_{2}\right|, \quad \forall z_{1}, z_{2}, \hat{z} \in \mathbb{R}, k \geq 0,
\end{aligned}
$$


where $N_{k}$ is a bounded function satisfying

$$
\int_{0}^{+\infty} N_{k} d k<+\infty
$$

Proof We suppose that $Z_{k}$ and $\hat{Z}_{k}$ are two solutions of (3) with different initial states $\varphi(k)$ and $\psi(k)(k \in[-\tau, 0])$, respectively. That is,

$$
\left\{\begin{aligned}
d Z_{k}= & h\left(k, Z_{k}, Z_{k-\tau}\right) d k+p\left(k, Z_{k}, Z_{k-\tau}\right) d C_{k} & & \\
& +q\left(k, Z_{k}, Z_{k-\tau}\right) d V_{k}, & & k \in[0,+\infty), \\
Z_{k}(\gamma) & =\varphi(k), & & k \in[-\tau, 0],
\end{aligned}\right.
$$

and

$$
\left\{\begin{array}{rlrl}
d \hat{Z}_{k}= & h\left(k, \hat{Z}_{k}, \hat{Z}_{k-\tau}\right) d k+p\left(k, \hat{Z}_{k}, \hat{Z}_{k-\tau}\right) d C_{k} & & \\
& +q\left(k, \hat{Z}_{k}, \hat{Z}_{k-\tau}\right) d V_{k}, & & k \in[0,+\infty), \\
\hat{Z}_{k}(\gamma)=\varphi(k), & & k \in[-\tau, 0] .
\end{array}\right.
$$

Then, for a Lipschitz continuous sample $C_{k}(\gamma)$ and $V_{k}(\gamma)$, it holds that

$$
\left\{\begin{aligned}
Z_{k}(\gamma)= & Z_{0}+\int_{0}^{k} h\left(r, Z_{r}(\gamma), Z_{r-\tau}(\gamma)\right) d r & & \\
& +\int_{0}^{k} p\left(r, Z_{r}(\gamma), Z_{r-\tau}(\gamma)\right) d C_{r}(\gamma) & & \\
& +\int_{0}^{k} q\left(r, Z_{r}(\gamma), Z_{r-\tau}(\gamma)\right) d V_{r}(\gamma), & & k \in[0,+\infty), \\
Z_{k}(\gamma)= & \varphi(k), & & k \in[-\tau, 0],
\end{aligned}\right.
$$

and

$$
\left\{\begin{aligned}
\hat{Z}_{k}(\gamma)= & \hat{Z}_{0}+\int_{0}^{k} h\left(r, \hat{Z}_{r}(\gamma), \hat{Z}_{r-\tau}(\gamma)\right) d r & & \\
& +\int_{0}^{k} p\left(r, \hat{Z}_{r}(\gamma), \hat{Z}_{r-\tau}(\gamma)\right) d C_{r}(\gamma) & & \\
& +\int_{0}^{k} q\left(r, \hat{Z}_{r}(\gamma), \hat{Z}_{r-\tau}(\gamma)\right) d V_{r}(\gamma), & & k \in[0,+\infty), \\
\hat{Z}_{k}(\gamma)= & \varphi(k), & & k \in[-\tau, 0] .
\end{aligned}\right.
$$

By condition (22), Lemma 4.1 in [9], and Theorem 3.2 in [27], we have

$$
\begin{aligned}
\mid Z_{k}(\gamma) & -\hat{Z}_{k}(\gamma) \mid \\
\leq & \mid Z_{0}-\hat{Z}_{0}+\int_{0}^{k} h\left(r, Z_{r}(\gamma), Z_{r-\tau}(\gamma)\right)-h\left(r, \hat{Z}_{r}(\gamma), \hat{Z}_{r-\tau}(\gamma)\right) d r \\
& +\int_{0}^{k} p\left(r, Z_{r}(\gamma), Z_{r-\tau}(\gamma)\right)-p\left(r, \hat{Z}_{r}(\gamma), \hat{Z}_{r-\tau}(\gamma)\right) d C_{r}(\gamma) \\
& +\int_{0}^{k} q\left(r, Z_{r}(\gamma), Z_{r-\tau}(\gamma)\right)-q\left(r, \hat{Z}_{r}(\gamma), \hat{Z}_{r-\tau}(\gamma)\right) d V_{r}(\gamma) \mid \\
\leq & \left|Z_{0}-\hat{Z}_{0}\right|+\left|\int_{0}^{k} h\left(r, Z_{r}(\gamma), Z_{r-\tau}(\gamma)\right)-h\left(r, \hat{Z}_{r}(\gamma), \hat{Z}_{r-\tau}(\gamma)\right) d r\right|
\end{aligned}
$$




$$
\begin{aligned}
& +\left|\int_{0}^{k} p\left(r, Z_{r}(\gamma), Z_{r-\tau}(\gamma)\right)-p\left(r, \hat{Z}_{r}(\gamma), \hat{Z}_{r-\tau}(\gamma)\right) d C_{r}(\gamma)\right| \\
& +\left|\int_{0}^{k} q\left(r, Z_{r}(\gamma), Z_{r-\tau}(\gamma)\right)-q\left(r, \hat{Z}_{r}(\gamma), \hat{Z}_{r-\tau}(\gamma)\right) d V_{r}(\gamma)\right| \\
& \leq\left|Z_{0}-\hat{Z}_{0}\right|+\int_{0}^{k} N_{r}\left|Z_{r}(\gamma)-\hat{Z}_{r}(\gamma)\right| d r \\
& \quad+K(\gamma) \int_{0}^{k} N_{r}\left|Z_{r}(\gamma)-\hat{Z}_{r}(\gamma)\right| d r+\int_{0}^{k} N_{r}\left|Z_{r}(\gamma)-\hat{Z}_{r}(\gamma)\right| d r \\
& =\left|Z_{0}-\hat{Z}_{0}\right|+\int_{0}^{k}(2+K(\gamma)) N_{r}\left|Z_{r}(\gamma)-\hat{Z}_{r}(\gamma)\right| d r,
\end{aligned}
$$

where $K(\gamma)$ is the Lipschitz constant of $C_{k}(\gamma)$.

According to Gronwall's inequality, we have

$$
\begin{aligned}
\left|Z_{k}(\gamma)-\hat{Z}_{k}(\gamma)\right| & \leq\left|Z_{0}-\hat{Z}_{0}\right| \exp \left(\left(2+K(\gamma) \int_{0}^{k} N_{r} d r\right)\right. \\
& \leq\left|Z_{0}-\hat{Z}_{0}\right| \exp \left((2+K(\gamma)) \int_{0}^{+\infty} N_{r} d r\right) \\
& \leq \sup _{r \in[-\tau, 0]}\left|Z_{r}-\hat{Z}_{r}\right| \exp \left((2+K(\gamma)) \int_{0}^{+\infty} N_{r} d r\right), \quad \forall k>0 .
\end{aligned}
$$

Thus we have

$$
\left|Z_{k}(\gamma)-\hat{Z}_{k}(\gamma)\right| \leq \sup _{r \in[-\tau, 0]}\left|Z_{r}-\hat{Z}_{r}\right| \exp \left((2+K(\gamma)) \int_{0}^{+\infty} N_{r} d r\right), \quad \forall k>0 .
$$

Thus, by Theorem 2 in [10], we have

$$
\lim _{x \rightarrow+\infty} \mathcal{M}\{\gamma \in \Gamma \mid K(\gamma) \leq x\}=1
$$

Then there exists a positive number $H$ such that

$$
\mathcal{M}\{\gamma \in \Gamma \mid K(\gamma) \leq H\} \geq 1-\varepsilon
$$

for any given $\epsilon>0$. Because

$$
\int_{0}^{+\infty} N_{s} d s<+\infty
$$

take

$$
\delta=\exp \left(-(2+K(\gamma)) \int_{0}^{+\infty} N_{r} d r\right) \epsilon
$$

Then $\left|Z_{k}(\gamma)-\hat{Z}_{k}(\gamma)\right| \leq \epsilon$ provided that

$$
\sup _{r \in[-\tau, 0]}\left|Z_{r}-\hat{Z}_{r}\right| \leq \delta
$$

and $K(\gamma) \leq H$. 
Hence,we have $\left|Z_{k}-\hat{Z}_{k}\right| \rightarrow 0$ as long as $\sup _{r \in[-\tau, 0]}\left|Z_{r}-\hat{Z}_{r}\right| \rightarrow 0$, which implies that

$$
\mathcal{M}\left\{\left|Z_{k}(\gamma)-\hat{Z}_{k}(\gamma)\right| \leq \epsilon\right\}=1-\varepsilon, \quad \forall k>0
$$

In other words,

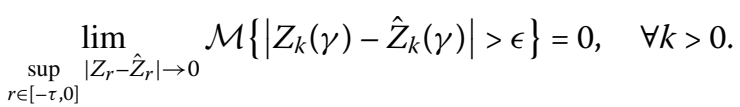

So the uncertain delay differential equation with $V$-jump (3) is stable in measure according to Definition 5. This completes the proof.

Corollary 1 Supposing that $u_{i k}, v_{i k}$, and $\eta_{i k}(i=1,2,3)$ are real-valued functions, the linear uncertain delay differential equations with $V$-jump

$$
\begin{aligned}
d Z_{k}= & \left(u_{1 k} Z_{k}+v_{1 k} Z_{k-\tau}+\eta_{1 k}\right) d k+\left(u_{2 k} Z_{k}+v_{2 k} Z_{k-\tau}+\eta_{2 k}\right) d C_{k} \\
& +\left(u_{3 k} Z_{k}+v_{3 k} Z_{k-\tau}+\eta_{3 k}\right) d V_{k}
\end{aligned}
$$

is stable in measure if $u_{i k}, v_{i k}$, and $\eta_{i k}(i=1,2,3)$ are bounded and satisfy

$$
\int_{0}^{+\infty} u_{1 k} d k<+\infty
$$

and

$$
\int_{0}^{+\infty} u_{2 k} d k<+\infty
$$

and

$$
\int_{0}^{+\infty} u_{3 k} d k<+\infty
$$

Proof Take $h(k, z, \hat{z})=u_{1 k} z+v_{1 k} \hat{z}+\eta_{1 k}, p(k, z, \hat{z})=u_{2 k} z+v_{2 k} \hat{z}+\eta_{2 k}$, and $q(k, z, \hat{z})=u_{3 k} z+$ $v_{3 k} \hat{z}+\eta_{3 k}$. Let $Q$ denote a common upper bound of $\left|u_{i k}\right|,\left|v_{i k}\right|$, and $\left|\eta_{i k}\right|(i=1,2,3)$. The inequalities

$$
|h(k, z, \hat{z})| \vee|p(k, z, \hat{z})| \vee|q(k, z, \hat{z})| \leq Q(1+|z|+|\hat{z}|)
$$

and

$$
\begin{aligned}
& \left|h\left(k, z_{1}, \hat{z}\right)-h\left(k, z_{2}, \hat{z}\right)\right| \vee\left|p\left(k, z_{1}, \hat{z}\right)-p\left(k, z_{2}, \hat{z}\right)\right| \vee\left|q\left(k, z_{1}, \hat{z}\right)-q\left(k, z_{2}, \hat{z}\right)\right| \\
& \quad \leq\left(u_{1 k} \vee u_{2 k} \vee u_{3 k}\right)\left|z_{1}-z_{2}\right| \leq Q\left|z_{1}-z_{2}\right|
\end{aligned}
$$

hold.

According to Theorem 2, we have that the linear uncertain delay differential equation with $V$-jump (27) with initial states has a unique solution. Since

$$
\begin{aligned}
& \left|h\left(k, z_{1}, \hat{z}\right)-h\left(k, z_{2}, \hat{z}\right)\right| \vee\left|p\left(k, z_{1}, \hat{z}\right)-p\left(k, z_{2}, \hat{z}\right)\right| \vee\left|q\left(k, z_{1}, \hat{z}\right)-q\left(k, z_{2}, \hat{z}\right)\right| \\
& \quad \leq\left(u_{1 k} \vee u_{2 k} \vee u_{3 k}\right)\left|z_{1}-z_{2}\right|,
\end{aligned}
$$


we take $N_{k}=u_{1 k} \vee u_{2 k}$, which is integrable on $[0,+\infty)$, since we have

$$
\int_{0}^{+\infty} u_{1 k} d k<+\infty
$$

and

$$
\int_{0}^{+\infty} u_{2 k} d k<+\infty
$$

and

$$
\int_{0}^{+\infty} u_{3 k} d k<+\infty
$$

By using Theorem 3, the linear uncertain delay differential equation with $V$-jump (27) is stable in measure.

\subsection{Some examples}

Example 1 Consider an uncertain delay differential equation with $V$-jump

$$
d Z_{k}=a d k+b Z_{k-\tau} d C_{k}+c d V_{k}
$$

Obviously, the coefficients $h(k, z, \hat{z})=a, p(k, z, \hat{z})=b \hat{z}$, and $q(k, z, \hat{z})=c$ are one-sided local Lipschitz continuous.

In addition,

$$
\begin{aligned}
& |h(k, z, \hat{z})| \vee|p(k, z, \hat{z})| \vee|q(k, z, \hat{z})| \\
& \quad=|a| \vee|b \hat{z}| \vee|c| \\
& \quad=\max (|a|,|b|,|c|)(1+|z|+|\hat{z}|), \quad \forall z, \hat{z} \in \mathbb{R}, k \in[0, T] .
\end{aligned}
$$

By using Theorem 2, it has a unique continuous solution.

In fact, the analytical solution of $d Z_{k}=a d k+b Z_{k-\tau} d C_{k}+c d V_{k}$ with the initial states $\phi(k)(k \in[-\tau, 0])$ is

$$
Z_{k}= \begin{cases}\phi(k), & k \in[-\tau, 0], \\ \phi(0)+a k+b \int_{0}^{k} \phi(r-\tau) d C_{r}+c V_{k}, & k \in(0, \tau], \\ Z_{\tau}+a(k-\tau)+b \int_{\tau}^{k} Z_{r-\tau} d C_{r}+c\left(V_{k}-V_{\tau}\right), & k \in(\tau, 2 \tau], \\ \cdots \cdots & \end{cases}
$$

Example 2 Consider an uncertain delay differential equation with $V$-jump

$$
d Z_{k}=a d k+b d C_{k}+c Z_{k-\tau} d V_{k} .
$$

Obviously, the coefficients $h(k, z, \hat{z})=a, p(k, z, \hat{z})=b$, and $q(k, z, \hat{z})=c \hat{z}$ are one-sided local Lipschitz continuous.

$$
\begin{aligned}
& |h(k, z, \hat{z})| \vee|p(k, z, \hat{z})| \vee|q(k, z, \hat{z})|=|a| \vee|b| \vee|c \hat{z}| \\
& \quad=\max (|a|,|b|,|c|)(1+|z|+|\hat{z}|), \quad \forall z, \hat{z} \in \mathbb{R}, k \in[0, T] .
\end{aligned}
$$


By using Theorem 2, it has a unique continuous solution.

In fact, the analytical solution of $d Z_{k}=a d k+b Z_{k-\tau} d C_{k}+c d V_{k}$ with the initial states $\phi(k)(k \in[-\tau, 0])$ is

$$
Z_{k}= \begin{cases}\phi(k), & k \in[-\tau, 0], \\ \phi(0)+a k+b C_{k}+c \int_{0}^{k} \phi(r-\tau) d V_{r}, & k \in(0, \tau], \\ Z_{\tau}+a(k-\tau)++b\left(C_{k}-C_{\tau}\right)+c \int_{\tau}^{k} Z_{r-\tau} d V_{r}, & k \in(\tau, 2 \tau] \\ \cdots \ldots & \end{cases}
$$

Example 3 Consider an uncertain delay differential equation with $V$-jump

$$
d Z_{k}=a Z_{k-\tau} d k+b Z_{k-\tau} d C_{k}+c Z_{k-\tau} d V_{k}
$$

Obviously, the coefficients $h(k, z, \hat{z})=a \hat{z}, p(k, z, \hat{z})=b \hat{z}$, and $q(k, z, \hat{z})=c \hat{z}$ are one-sided local Lipschitz continuous.

$$
\begin{aligned}
& |h(k, z, \hat{z})| \vee|p(k, z, \hat{z})| \vee|q(k, z, \hat{z})|=|a z| \vee|b \hat{z}| \vee|c \hat{z}| \\
& \quad=\max (|a|,|b|,|c|)(1+|z|+|\hat{z}|), \forall z, \hat{z} \in \mathbb{R}, k \in[0, T] .
\end{aligned}
$$

By using Theorem 2, it has a unique continuous solution.

In fact, the analytical solution of $d Z_{k}=a Z_{k-\tau} d k+b Z_{k-\tau} d C_{k}+c Z_{k-\tau} d V_{k}$ with the initial states $\phi(k)(k \in[-\tau, 0])$ is

$$
Z_{k}= \begin{cases}\phi(k), & k \in[-\tau, 0], \\ \phi(0)+a \int_{0}^{k} \phi(r-\tau) d r+b \int_{0}^{k} \phi(r-\tau) d C_{r} & \\ \quad+c \int_{0}^{k} \phi(r-\tau) d V_{r}, & k \in(0, \tau], \\ Z_{\tau}+a \int_{\tau}^{k} Z_{r-\tau} d r & \\ & +b \int_{\tau}^{k} Z_{r-\tau} d C_{r}+c \int_{\tau}^{t} Z_{r-\tau} d V_{r}, \quad k \in(\tau, 2 \tau], \\ \ldots & \end{cases}
$$

Example 4 Consider an uncertain delay differential equation with $V$-jump

$$
d Z_{k}=\left(\exp (-k) Z_{k-\tau}+\mu\right) d k+\sigma d C_{k}+v d C_{k}, \quad k \in[0,+\infty) .
$$

It follows from conditions that real-valued functions $\exp (-k),|\mu|,|\sigma|$, and $|\nu|$ are bounded on the interval $[0,+\infty)$. Since

$$
\int_{0}^{+\infty} \exp (-k) d k=1<+\infty
$$

according to Corollary 1 , the linear uncertain delay differential equation with $V$-jump (31) is stable in measure. 


\section{Conclusions}

In this paper, we propose uncertain delay differential equations with $V$-jump and establish the existence, uniqueness, and stability theorem of solution for the uncertain differential equations with $V$-jump. One source of weakness in our study of uncertain delay differential equations with $V$-jump is the lack of numerical methods and applications; these will be the focus of our future research.

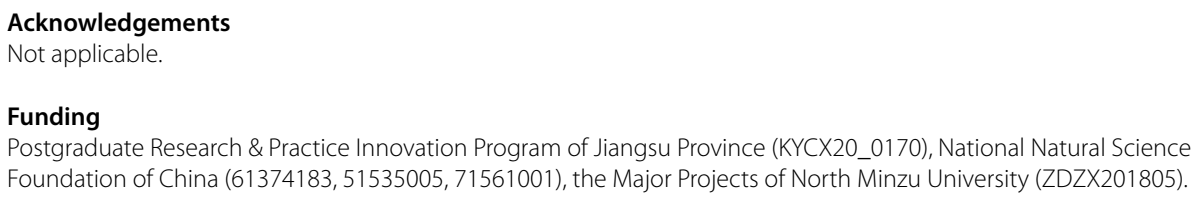

\section{Publisher's Note}

Springer Nature remains neutral with regard to jurisdictional claims in published maps and institutional affiliations.

Received: 26 March 2020 Accepted: 13 August 2020 Published online: 26 August 2020

\section{References}

1. Ito, K.: On stochastic differential equations. Mem. Am. Math. Soc. 4, 1-51 (1951)

2. Black, F., Scholes, M.: The pricing of option and corporate liabilities. J. Polit. Econ. 81, 637-654 (1973)

3. Chen, S., Li, X., Zhou, X.: Stochastic linear quadratic regulators with indefinite control weight costs. SIAM J. Control Optim. 36(5), 1685-1702 (1998)

4. Gray, A., Greenhalgh, D., Mao, X., Pan, J.: A stochastic differential equation SIS epidemic model. SIAM J. Appl. Math. 71, 876-902 (2011)

5. Ramachandran, K., Tsokos, C.: Stochastic differential games: theory and applications. Mol. Microbiol. 35(5), 961-973 (2012)

6. Liu, B.: Uncertainty Theory: A Branch of Mathematics for Modeling Human Uncertainty. Springer, Berlin (2010)

7. Liu, B.: Fuzzy process, hybrid process and uncertain process. J. Uncertain Syst. 2(1), 3-16 (2008)

8. Liu, B.: Some research problems in uncertainty theory. J. Uncertain Syst. 1, 3-10 (2009)

9. Chen, X., Liu, B.: Existence and uniqueness theorem for uncertain differential equations. Fuzzy Optim. Decis. Mak. 9(1), 69-81 (2010)

10. Yao, K., Gao, J., Gao, Y.: Some stability theorems of uncertain differential equation. Fuzzy Optim. Decis. Mak. 12(1), 3-13 (2013)

11. Liu, H., Ke, H., Fei, W.: Almost sure stability for uncertain differential equation. Fuzzy Optim. Decis. Mak. 13(4), 463-473 (2014)

12. Sheng, Y., Wang, C.: Stability in p-th moment for uncertain differential equation. J. Intell. Fuzzy Syst. 26(3), 1263-1271 (2014)

13. Yao, K., Ke, H., Sheng, Y.: Stability in mean for uncertain differential equation. Fuzzy Optim. Decis. Mak. 14(3), 365-379 (2015)

14. Sheng, Y., Gao, J.: Exponential stability of uncertain differential equation. Soft Comput. 20, 3673-3678 (2016)

15. Yang, X., Ni, Y., Zhang, Y.: Stability in inverse distribution for uncertain differential equations. J. Intell. Fuzzy Syst. 32(3), 2051-2059 (2017)

16. Yao, K., Chen, X.: A numerical method for solving uncertain differential equations. J. Intell. Fuzzy Syst. 25(3), 825-832 (2013)

17. Zhu, Y.: Uncertain optimal control with application to a portfolio selection model. Cybern. Syst. 41(7), 535-547 (2010)

18. Yang, X., Gao, J.: Uncertain differential games with application to capitalism. J. Uncertain. Anal. Appl. 1(17), 1-11 (2013)

19. Yang, X., Gao, J.: Linear-quadratic uncertain differential games with application to resource extraction problem. IEEE Trans. Fuzzy Syst. 24(4), 819-826 (2016) 
20. Gao, R.: Uncertain wave equation with infinite half-boundary. Appl. Math. Comput. 304, $28-40$ (2017)

21. Gao, R., Ralescu, D.A.: Uncertain wave equation for vibrating string. IEEE Trans. Fuzzy Syst. 27(7), 1323-1331 (2019)

22. Gao, R., Ma, N., Sun, G.: Stability of solution for uncertain wave equation. Appl. Math. Comput. 365, 469-478 (2019)

23. Gao, R., Liu, K., Li, Z.: American barrier option pricing formulas for stock model in uncertain environment. IEEE Access 7, 97846-97856 (2019)

24. Lu, Q., Zhu, Y., Lu, Z.: Uncertain fractional forward difference equations for Riemann-Liouville type. Adv. Differ. Equ. 2019, 147 (2019)

25. Yao, K.: Uncertainty Differential Equation. Springer, Berlin (2016)

26. Deng, L., Zhu, Y.: Uncertain optimal control with jump. ICIC Express Lett. 3(2), 419-424 (2012)

27. Deng, L., Zhu, Y.: Existence and uniqueness theorem of solution for uncertain differential equations with jump. ICIC Express Lett. 6(10), 2693-2698 (2012)

28. Deng, L.: Multidimensional uncertain optimal control of linear quadratic models with jump. J. Comput. Inf. Syst. 8(18), 7441-7448 (2012)

29. Deng, L., Zhu, Y.: An uncertain optimal control model with $n$ jumps and application. Comput. Sci. Inf. Syst. 9(4), 1453-1468 (2012)

30. Deng, L., Zhu, Y.: Uncertain optimal control of linear quadratic models with jump. Math. Comput. Model. 57, 2432-2441 (2013)

31. Deng, L., Chen, Y.: Optimistic value model of uncertain linear quadratic optimal control with jump. J. Adv. Comput. Intell. Intell. Inform. 20(2), 189-196 (2016)

32. Deng, L., Chen, Y.: Optimal control of uncertain systems with jump under optimistic value criterion. Eur. J. Control 38, 7-15 (2017)

33. Deng, L., You, Z., Chen, Y.: Optimistic value model of multidimensional uncertain optimal control with jump. Eur. J. Control 39, 1-7 (2018)

34. Barbacioru, I.: Uncertainty functional differential equations for finance. Surv. Math. Appl. 5, 275-284 (2010)

35. Ge, X., Zhu, Y.: Existence and uniqueness theorem for uncertain delay differential equations. J. Comput. Inf. Syst. 8(20), 41-83 (2012)

36. Wang, X., Ning, Y.: Stability of uncertain delay differential equations. J. Intell. Fuzzy Syst. 32(3), 2655-2664 (2017)

37. Wang, X., Ning, Y.: A new stability analysis of uncertain delay differential equations. Math. Probl. Eng. 2019, Article ID 1257386 (2019)

38. Jia, L., Sheng, Y.: Stability in distribution for uncertain delay differential equation. Appl. Math. Comput. 343, 49-56 (2019)

39. Gronwall, T.: Note on the derivatives with respect to a parameter of the solutions of a system of differential equations Ann. Math. 20(4), 292-296 (1919)

\section{Submit your manuscript to a SpringerOpen ${ }^{\circ}$ journal and benefit from:}

- Convenient online submission

- Rigorous peer review

- Open access: articles freely available online

- High visibility within the field

- Retaining the copyright to your article

Submit your next manuscript at $>$ springeropen.com 